\title{
LA DOCUMENTACIÓ DEL RETAULE DE SANTA MARIA DE SALAS
}

\author{
ANTONI JOSÉ I PITARCH \\ Universitat de Barcelona
}

El retaule de plata que fou de l'església de Santa Maria de Salas (Osca) és un dels pocs retaules d'argenteria conservats a l'antiga Corona d'Aragó i, juntament amb el de la seu de Girona són les úniques obres que permeten conèixer les característiques materials, tècniques i de formes que els argenters valencians i catalans desenrotllaren durant els dos primers terços i escaig del segle XIV, en paral·lel a les que posaven en pràctica, a la manera italiana, els pintors, a la manera més del nord de França els escultors, així com, també, els mestres vitrallers. L'escassetat però d'obres d'argenteria en relleu i volum, així com les diferències entre les obres conservades no permet una classificació-filiació per a aquests tipus de treballs, que resulta més possible quan es tracta de les plaques d'esmalt.

No és el propòsit d'aquestes pàgines indagar sobre la filiació del retaule de Santa Maria de Salas. La intenció és estrictament documental, en facilitar alguns documents inèdits totalment, per bé que en alguns casos el seu contingut era ja conegut, i en posar al dia les notícies d'arxiu referents al retaule per tal de configurar un primer corpus documental ordenat.

Dels autors que han escrit indirectament o directa sobre el retaule de Santa Maria de Salas n'hi ha tres que són els qui han fet aportacions documentals.

"Anuario de Isstudios Medievales". 29 (1999) 
En primer lloc Francisco Diego de Aynsa' qui donà a conèixer la inscripció conservada al dors dels set compartiments del retaule, explicativa de la història del mateix, les causes que provocaren que el rei Pere s'endugués del Santuari de Salas una quantitat d'argent (la guerra contra Castella), i el compromís del rei de manar fer un retaule de plata en esmena de la que s'havia endut ${ }^{2}$. Es tracta d'una autèntica inscripció que sintetitza la història del retaule.

Però no fou fins el $1946^{3}$ quan del Arco donà a conèixer, en un estudi sobre el Santuari de Santa Maria de Salas, la major part de les notícies que teniem sobre el retaule. A partir de la documentació de l'Arxiu de la Corona d'Aragó, tot sense citar-ne la procedència exacta en la major part de les notícies (o sense citar-ne cap), aquest autor, llegint alguns registres de la Cancelleria Reial i alguns volums del Mestre Racional, lligà per primera vegada la petita història del retaule. No s'acaba d'entendre massa com, amb la consulta que en féu de les fonts, no les donés o no estirés el fil per tal de deixar més clarificades les qüestions referents a la realització del retaule.

\footnotetext{
'AYNSA (1619), p. 594

${ }^{2}$ Es coneixen uns documents referents a les incautacions de tresors d'esglésies que el rei féu amb motiu de la guerra contra Castella. Serveixin com exemples un passatge de la Crònica del rei Pere el Cerimoniós, referit a la catedral de València i dos documents, també de la catedral de de València:

a.- [1364, Juny, 15-24. València]. Pere el Ceremoniós pren vaixella d'or i de plata de la Seu de València per realitzar els pagaments als soldats, amb motiu de la guerra contra Castella. Crónica de Pere el Cerimoniós a "Les quatre grans Cròniques". Revisió del text, pròlegs i notes per Ferran Soldevila. Editorial Selecta, Barcelona, 1971, Capítol sisè, 45, pàg. 1144: (45)... "E, passada la fortuna, lo dit rei de Castella tornà-se' $n$ e féu reverència a l'esglesia de Madona Santa Maria, ab un dogal al coll e en camisa e en bragues, feent-li gràcies com era estort de perill de la fortuna dessús dita. E nos tornam a València a quinze dies del mes de juny aprés sigüent. E, esdevenc-se que, per fer paga a la gent de nostre estol, haguem gran fretura de moneda, e los soldats nostres sens diners no podien retre llur deute als afers de la guerra. Per què $a b$ llicència del bisbe, plevim-nos de totes les vaixelles de les esgleies, així com són reetaules d'argent, creus, calzes e llànties, e encensers, e tota altra vaixella d'or e d'argent que trobam en los reliquiaris de les dites esgleies. E aquella prenguem e distribuïm entre los nostres soldats. E feta estima de la de la dita vaixella d'argent e d'or de què ens érem plevits clels dits reliquiaris de les dites esglesies, fem satisfacció e esmena complida dins breu temps aprés següent."

b.- Inventari dels objectes preuats que la seu i les esglésies de València entregaren al rei Pere el Cerimoniós el dia 10 de maig de 1364. ACA, Canc., reg. 1188, fol. 178. Pub. per José Sanchis Sivera a La esmalteria valenciana en la Edad Media. AÀV, núm. VII, 1921, pàgs. 3-50 (31-35).

c.- 1367 , gener, 4. Barcelona. El rei Pere reconeix que el bisbe de València, Vidal de Blanes, i el Capítol de la seu valenciana, per fer front a la guerra contra Castella li deixaren perles $\mathrm{i}$ altres pedres precioses per valor de 1000 florins d'or, així com diverses joies i vasos de plata valorats segons que en document públic consta. ACA, Canc., reg. 1344, fol. 59.

${ }^{3}$ DEL ARCO (1946), pàgs. 117-119
} 
Amb tot, devem a del Arco la primera agrupació de notícies que confirmen la inscripció del darrere del retaule i que posen nous coneixements de persones i fets. A partir d'aquest autor sabem qui fou el mestre de les set escenes del retaule, l'argenter Bartomeu Tutxó; com s'arbitrà el finançament de l'obra per part del rei en dirigir cap al retaule de dècima bienal del bisbat d'Osca que el papa li havia atorgat; les persones encarregades de recaptar la dècima, els canonges Ramon Vigorós i Pere Oliver; els pagaments, amb problemes o sense problemes, a l'argenter; i els noms dels canviadors de Barcelona, Eimeric Dusay i Jaume de Gualbes, a la taula dels quals havien estat dipositades algunes sumes per poder pagar a Tutxó. Totes aquestes notícies, citades, transcrites parcialment o senceres han estat recuperades (llevat d'algunes que no hi ha hagut manera de trobar) i s'han transcrit íntegrament en aquest treball.

D'altra banda, després de del Arco cal esperar l'article-fitxa del catàleg de l'exposició “Signos" (1933), realitzat per Esteban", per trobar noves referències sobre el retaule de Santa Maria de Salas, extretes en aquest cas de l'Arxiu de la Catedral d'Osca (ACH), i facilitades per don Antonio Gudiol. Aquestes noves referències proporcionen més informació sobre la $\mathrm{col} \cdot$ lecta de la dècima i el nomenament del col-lectors, sobre l'incompliment dels col-lectors en l'entrega dels diners per obrar el retaule, i la data d'entrega del retaule al bisbe d'Osca per a la festa de Sant Joan Baptista del 1367 (24 de juny). Malhauradament, (la publicació no ho permetia), no han estat publicats els documents al respecte i prenem com a referència les cites i les fonts que Esteban dóna.

Ara, aplegades les informacions i documents esmentats, publicats parcialment i sencers, amb o sense referència d'arxiu, es publiquen de manera íntegra els que es conserven a l'Arxiu de la Corona d'Aragó (llevat d'un que no s'ha trobat), i, per primera vegada, es publica el contracte del retaule de Santa Maria de Salas, inèdit quant a coneixement del mateix que porta la data de 15 de desembre de 1366, és a dir, vint mesos després de la data del 23 d'abril de 1364, quan el rei es comprometé a fer obrar un retaule amb les històries de la vida de la Verge, en esmena per haver pres les llànties i altres objectes de plata del Santuari de Santa Maria de Salas, i setze mesos més tard que l'argenter Bartomeu Tutxó hagués rebut un pagament de 3000 sous barcelonesos. Aquesta demora en el temps en signar les capitula-

${ }^{4}$ ESTEBAN (1993), pp. 400 i 402 
cions cal entendre-la com actitud de prudència per part de l'argenter en veure les dificultats que hi havia per abonar-li les quantitats assignades.

$\mathrm{Al}$ contracte queden reflectits els següents punts:

1. Bartomeu Tutxó rebrà 3000 sous per comprar argent per l'obra del retaule. (L'anotació que diu "de present" cal entendre-la amb data del 16 d'octubre de 1366).

2. Altres 700 sous seran dipositats a la taula d'Eimeric Dusay i Jaume de Gualbes, canviadors de Barcelona, quan el retaule serà acabat.

3. El canonge Pere Oliver, col-lector de la dècima, mentre duri l'obra del retaule, no percebrà res de la dècima.

4. Si durant la realització del retaule Bartomeu Tutxó necessita més argent que li'n puguin donar fins a 4000 sous més (a més dels 7000 sous anteriors).

5. Es fixa el termini d'entrega del retaule el 24 de juny de 1367 , festivitat de Sant Joan Baptista. (Per tant, el retaule s'havia d'obrar en mig any, temps que podríem considerar molt reduït, però que és indicador de la potència de l'obrador de Tutxó a Barcelona que, al mateix temps, treballava en altres encàrrecs).

6. El preu estipulat per marc d'argent treballat és de 6 lliures i 18 sous barcelonesos. (Aquesta anotació de moneda barcelonesa, com la de les quantitats anteriorment assenyalades és interessant i important retenir-la pels problemes que tingué el Mestre Racional en passar comptes).

7. El retaule ha de contenir la història dels set Goigs de la Verge. (Queda per tant fora de dubte que les escenes conservades són la totalitat del retaule).

8. El treball de l'argenter ha de ser d'un bon repussat, amb figures ben treballades i ben pensades.

9. L'argenter haurà de pagar tots els materials que entrin en l'obra del retaule, amb excepció de la fusta sobre la que es muntaran les escenes.

10. Quan el retaule sigui acabat Tutxó l'haurà d'entregar al canonge Oliver o a qui ell designi.

Així doncs, el contracte, que se'n fa ressò de la història precedent, deixa molt clar el preu, el termini, la temàtica i la tècnica d'execució del retaule, tot i que no es pot dir que tots els pactes foren complits al peu de la lletra. Si més no, el pagament dels 7.000 sous a l'argenter s'enderrerí fins l'1 de març de 1368. Precisament, el document d'aquesta data fa constar el pes del retaule una vegada acabat: 94 marcs, 6 unces i 3 ternals i mig de plata. 
El contracte és el document més important d'aquest petit corpus de notícies sobre el retaule de Santa Maria de Salas. Una vegada conegut no resta cap problema sobre la cronologia de l'obra ni sobre el nombre d'escenes que tingué el retaule al seu origen i que encara es conserven. Amb tot, com ja hem vist, la història del retaule continuà més enllà del Sant Joan de 1367. Catorze anys després, el 4 de juny de 1381, en passar comptes del retaule de Santa Maria de Salas, entre els fochs consten el pagament del viatge a Sant Mateu d'un home perquè el rei assignés mitjans pel retaule; el problema del pagament en sous jaquesos o barcelonesos per part dels collectors de la dècima; i sobre altres qüestions vinculables a pagaments per part de Pere Desvall i d'altres persones. 


\section{DOCUMENTS}

1364, abril, 23. Sant Mateu.

Lletra del rei Pere on fa constar que per causes de la guerra amb Castella es va veure obligat a prendre del santuari de Santa Maria de Salas llànties $i$ altres objectes de plata per valor de dos cents marcs de plata. Per esmenar aquesta incautació promet pagar al Capítol de la seu d'Osca 700 lliures jaqueses en les persones del Canonge Pere Oliver $i$ de l'oficial Ramon Vigoros els quals hauran de portar aquesta quantitat a Barcelona per fer un retaule amb històries de la vida de la Verge, destinat a Santa Maria de Salas. El retaule del que el rei es compromet, també, a pagar la mà d'obra, hauria de portar els escuts, coneguts pel canonge $i$ l'oficial esmentat, dels qui havien ofert les llànties incautades. Per fer front a les despeses, el rei atorga a l'obra del retaule les dècimes del bisbat d'Osca que el Papa li havia concedit, al temps que la meitat de les deixes pies del bisbat d'Osca.

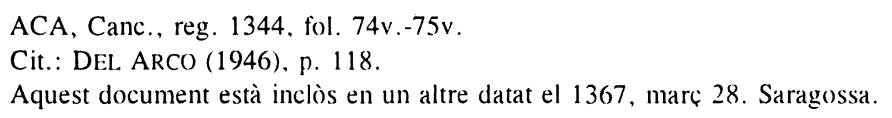

Nos Petrus etc. Dudum ordinationem et mandatum fecisse recolimus cum litera nostra tenoris sequentis. Nos en Pere per la gracia de deu Rey d' Arago etc. Attenents que nos per la necessitat gran en que som per la guerra havem fet pendre de la esgleya de nostra dona santa Maria de Sales de la ciutat d'Oscha doents marchs d'argent en lantes e en altres diverses coses lo qual argent fem pendre per mossen Blasco Aznarez de Borau, batle general d'Arago qui aquell argent per nom e per part nostra reebe. On volents fer deguda satisfacció e esmena del dit argent a la dita esgleya per ço per tenor de present confessam deure los dits doents marchs d'argent a nos amats nostres lo Capitol de la esgleya d'Osca en nom de la esgleya de Santa Maria de Sales ço es per lo preu de cascun march a raho de LXX solidos jaccenses lo march e jassia no sia estat venut si no a raho de sexanta cinc solidos lo march 
pero aytant valia si no que per la cuyta que nos haviem de vende se hague a vendre a menys falliment. Per que prometem en nostra bona fe Reyal de pagar e fer pagar a vos dit Capitol en nom de la dita esgleya septingentas libras jaccenses a la qual quantitat monta lo dit argent al dit for. Manants ab aquesta lletra al amat conseller e tresorer nostre micer Bernat d'Olzinelles cavaller e a son lochtinent que encontinent vos paguen la quantitat damunt dita en aquesta forma, es a saber que per nom e per part vostra e de la dita esgleya la reeba en Pere Oliver canonge e en Ramon Vigoros oficial d'Oscha, los quals sien tenguts fer sagrament e homenatge de portar o fer portar la dita quantitat en Barchinona e fer d'aquesta fer un reetaule en honor de nostra dona Santa Maria de Sales istoriat de la sua vida, les faedures del qual reetaule nos prometem de pagar aytant com costaran, axi que ultra la valor del dit argent nos vos atorgam de pagar les faedures'. E volem que en lo dit reetaule sien fets los senyals de tots aquells qui havien dades les lantees e altres joyes segons que ço saben los dits Pere Oliver e oficial pero quel dit reetaule haien a fer fer los damunt dits a ordinacio e consell d'en Pere dez Vall de la tresoreria nostra al qual nos havem comanat que ordon lo dit reetaule segons la nostra volentat. E per tal quel damunt dit fet haia mellor compliment e que axi cau se cove e fer se deu lo dit reetaule sia tost complit e acabat volem e manam que totes les decimes del bisbat d'Oscha sien assignades al dit deute axi que quantitat alguna de la dita decima no sia pagada a persona alguna sino als damunt dits en la forma dessus contenguda ans lo collidor qui huy es o lo subdelegat d'aquell facen sagrament $e$ homenatge als damunt dits Pere Oliver e oficial de respondrels de tota la quantitat que reebuda hauran pagats tansolament aquells D florins los quals en Bernat Badia capella e almoyner nostre nos presta e per nos liura al dit Pere dez Vall en nom del dit tresorer nostre. E haut compte de les dites decimes e pagats los dits D florins volem e manam que de totes les decimes sia respost als dits Pere Oliver e oficial per tal que torb algu no hi puxa venir. E volem que ells cullen les dites decimes e que a ells e no a altra persona ne sia respost. E manam als clergues e al Capitol de la esgleya d'Oscha que als dits canonge e oficial ne responen e no a altres persones com empero seran pagats los dits $\mathrm{D}$ florins prestats segons que [dit] es per lo dit en Bernat Badia pregam los dits canonge e Capitol d'Oscha e de Jacca e al abat de Montarago per ço que abans pusca esser acabat lo dit reetaule que les dites decimes vullen anticipar. E per tal quel dit reetaule sia abans complit ab aquesta matexa sospenem totes assignacions fetes sobre les dites decimes. E encara assignam la metat de tot ço que exira de les lexes pies del bisbat d'Oscha axi per composicio com encara per execucio. E que de la dita meytat sia respost als damunt dits Pere Oliver e oficial e quels comissaris deputats a reebre los diners de les dites lexes pies

\footnotetext{
'Segueix ratllat del qual reeteule nos prometem de pagar aytant com costaran axi que ultra la valor del dit argent.
} 
haien e sien tenguts de respondre de la dita meytat als dits Pere Oliver e official de d'aço los sien tenguts de fer sagrament e homenatge e si nou feien que los damuntdits Pere Oliver e oficial de lur propia autoritat puxen pendre tants dels dits bens que basten a la quantitat de la dita meytat no contrastants qual se vol assignacions per nos fetes o faedaores sots qual se vol forma de paraules. E per maior fermetat de totes coses damunt dites juram per nostre senyor deu e los sants quatre Evangelis de nos corporalment tocats e per nostra dona Santa Maria les demunt dites coses e cascuna d'aquelles attendre e fer attendre e complir e no contravenir per alguna raho. En testimoni dela qual cosa manam fer la present ab nostre segell secret esser segellada, dada en la vila de sent Matheu a XXIII dies d'abril en l'any dela nativitat de nostre senyor MCCCLXIIII. Rex Petrus. Cuius ordinationis et mandati pretextu procesum fuit quodammodo ad construccionem seu fabricacionem reetabuli antedicti si quia postea reperimus de jure talia que sunt ad dei servicium dedicata non debere fieri ad disposicionem seu consilium laycorum set pocius clericorum. Ideo predictam ordinacionem in eo quo cavetur in ea quod ad consilium dicti Petri de Vallo dictum reetabulum deberet fieri et quitquid ad consilium et ordinacionem ipsius et Petri Vincentii de Thesauraria nostra et alterius cuius cumque persone laycalis super eo factum extitit diutius huius serie revocandum. Mandantes expresse et de certa sciencia dictis Petro Oliverii et Raymundo Vigorosi ac Petro de Vallo et Petro Vincentii et aliis quibuslibet personis que in hoc intervenerint usque nunc seu debeant ammodo intervenire, quatenus de peccunia necessaria ad perfeccionem dicti reetabuli et de ipso etiam reetabulo cum perfectum fuerit repondeant Episcopo oscensi ad cuius disposicionem talia pertinere noscuntur aut cui voluerit eius loco quibuslibet mandatis verbo aut literatorie in contrarium factis et obligacionibus eciam fatis nostro Thesaurio aut aliis que et quas revocamus de certa sciencia et consulte obsistentibus nullo modo sit tamen per litera executoria nostri thesaurarii ut exibeatur super hoc quem nos per presentem mandamus magistro racionali curie nostre vel alii cuicumque a dictis Petro Oliverii, Raymundo Vigorosi, Petro de Vallo et Petro Vincentii et aliis solucionem vel soluciones inde facientibus compotum audito quod quitquid dicto Episcopo vel ad eius ordinacionem persolverint per modum supra expressum in construccione et perfeccione dicti reetabuli in eorum compoto admittere teneatur. Datum Cesarauguste XXVIII, die Marcii anno a nativitate domini M CCC LX VII. Rex Petrus.

[1364]

El capítol d'Osca esmerça 200 sous per aconseguir del rei Pere l'assignació de la quantitat necessària per obrar el retaule de Santa Maria de Salas. 
Pub. par.: DEI. ARCo (1946), pp. 117-118. Sense donar referència documental. Cfr.: Document de 1381, juny. 4. Saragossa.

“[...] per obs de fer les messions en perseguir que el senyor Rey fes assignacio del argent que havia pres de Santa Maria de Sales, los quals diners foren despeses per l'oficial ${ }^{\mathrm{a}}$ y per mi $\mathrm{i}^{\mathrm{b}}$ en dos viatges a Çaragoça hon lo senyor Rey hera, y un hom que trametem a Sent Mateu per haver la letra sobre la Decima [...]"

"el canonge Ramon Vigorós

${ }^{\mathrm{h}}$ el canonge Pere Oliver

1366, gener, 21. Barcelona.

El rei Pere comunica al bisbe d'Osca, Jimeno de Ribavellosa, el nomenament del canonge Pere Oliver subcol-lector a la diòcesi d'Osca de la dècima bienal de les rendes eclesiàstiques que el papa li havia atorgat.

ACH, Extravagantes-papel.

Cit.: Estriban (1993), p. 400)

4

1366, març, 12. Saragossa.

El rei Pere ven al bisbe d'Osca, Jimeno de Ribavellosa, els drets reials sobre els llocs pertanyents a la mitra d'Osca i a les seus d'Osca i de Jaca, pel preu de 15000 sous jaquesos.

$\mathrm{ACH}$, sign. 2-50

Cit.: ESTEBBAN (1993), pàg. 400 i 402 
1366, març, 15 .

El rei Pere comunica als subcol-lectors de la dècima bienal del bisbat d'Osca que ha ordenat al mestre racional l'entrega d'una quantitat destinada a obrar el retaule de Santa Maria de Salas.

ACH, Extravagantes-papel

Cit.: Esteban (1993). p. 402.

6

1366, agost, 24. Saragossa.

El rei Pere explica les causes que havien motivat la incautació de la plata de Santa Maria de Salas -la guerra amb Castella-i que, per esmena, havia ordenat fer un retaule de plata. Per a tal fi havia ordenat al seu tresorer entregar als canonges d'Osca Pere Oliver i Ramon Vigorós una quantitat que aquests havien de destinar al retaule a obrar a Barcelona. Tanmateix la quantitat atorgada pel rei el bisbe d'Osca. Jimeno de Ribavellosa, l'havia destinada a d'altres necessitats i hagué de recòrrer al rei per sol-licitar una pròrroga.

Pub. par.: ESTI:BAN (1993), p. 402. Sense donar referència documental.

[...] per urgent necessitat que haviem per la guerra de Castella, haviem despullada l'esgleya de Santa Maria de Sales de lantees e d'altres joyes d'argent... per descarrech de nostra consciencia haviem ordonat que fos fet un retaula d'argent en la dita esgleya $[\ldots]$

1366, octubre, 16. Barcelona.

Pagament de 3000 sous barcelonesos a Bartomeu Tutxó, argenter de Barcelona, pel retaule de Santa Maria de Salas.

ACA, RP, MR, vol. 1803, fol. 31 
Cit.: DEL. ARCO (1946), p. 118. Sense donar referència documental.

Item foren donats an Barthomeu Tutxo argenter dela ciutat de Barchanona per raho de I retaula d'argent que feu a Santa Maria de Sales. Et avi Iles apoques ço es la una feta e aclosa per en Barthomeu Gomar notari de Barchanona a XVI dies del mes d'octubre del any MCCCLXVI de quantitat del IIIm solidos de barchanona. E altra feta e aclosa per en Johan Martinez de Sixena notari lo primer dia del mes de març del any dela nativitat de nostre senyor M CCC LX VIII de quantitat de VIIIm solidos jaccenses ${ }^{2}$. E axi son en summa - Xm solidos barchinonenses qui fet cambi ab jaquesos a rao $\mathrm{de}^{3}$ XII diners barchinonesos mes per libra de jaquesos que valien en temps per ells foren tenguts fer lo qual retaule lo senyor Rey ab letra sua dada en Sant Mathey a XXIII dies del mes d'abril del any M CCC LXIIII mana esser fet per ops de la dita esgleya e que fos pagat dels diners de la dita decima, ço es d'aquelles DCC lliures jaqueses les quals ab la dita letra confessa deure al capitol de la esgleya d'Osca en nom de la esgleya de Santa Maria de Sales de la dita ciutat per satisffacció de CC marchs d'argent que per certes necessitats se havia preses de la dita esgleya e d'aquelles a pagar assigna los diners de la dita decima ab letra dessusdita ${ }^{4}$-IX m DXXIII solidos X diners jaccenses.

[Al marge:] Letra del senyor Rey ab la qual atorga deure al capitol dela seu d'Oscha per preu o valor de CC marchs d'argent a rao de CCC solidos jaccenses lo march los dits XIIII $m$ solidos jaccenses. I es cert que romanien a pagar dela dita quantitat IXm DCCLXVI sous II diners jaccenses. E jassie que axi no sien posats en data sino los dits IX mDXXIII solidos X diners jaccenses es cert quel senyor Rey en carta a mossen Gil Sanchez Munyoz bisbe d'Oscha CCCC florins d'or per esmena de les faedures del dit retaule. E axi fou cobrada la dita letra e apoques del dit retaule.

1366, octubre, 16. Barcelona.

Àpoca de 1000 sous (= 150 lliures) a favor de l'argenter Bartomeu Tutxó per raó del retaule que aquest va prometre obrar per a l'església de Santa Maria de Salas del bisbat d'Osca.

\footnotetext{
'Segueix ratllat barchinonenses.

${ }^{3}$ Segueix ratllat III.

${ }^{4}$ Segueix ratllat $\mathrm{Xm}$ solidos barchinonenses.
} 
Sit omnibus notum quod Ego Barholomeus Tutxo argenterius et civis Barchinone confiteor et recognosco vobis dilecto Petro Oliverii canonico oscensi presenti et vestris quod tradidistis et solvistis mihi ad meam voluntatem centum quinquaginta libras monete Barchinone de terno quas michi bistraxistis pro ac racdione cuiusdam Retrotabuli argenti quod operari teneor atque promisi ad opus Ecclesie beate Marie de Salis Episcopatu oscensi. Et ideo renunciando excepcione pecunie non numerate et non habite et non recepte et doli. In testimonium omnium premissorum iubeo inde vobis fieri presens per notarium infrascriptum. Actum est hoc Barchinone sextadecima die octobris anno a Nativitate domini Millessimo trecentesimo sexagesimo sexto. Sig + num Bartholome Tutxo predicti qui hoc laudo et firmo. Testes huius rei sunt Periconus Çestrada et Periconus Costa cives Barchinone. Sig + num Bartholomei Gomar, auctoritate Regia notarii publici Barchinone, qui hoc scripsit et clausit.

[Verso:] Apocha dels $\mathrm{M}$ sous donats an Tutxo argenter.

1366, novembre, 5. Barcelona.

El rei Pere, estranyat de que Pere Oliver i Ramon Vigorós no hagin complert amb la paraula donada, els adverteix que seran objecte de la seva indignació si en el termini de vuit dies no entregaven la recaptació de la dècima al seu tresorer de Barcelona. Aquest endarreriment no permet al rei l'ofrena del retaule a Santa Maria de Salas en el termini que havia promès fer-ho.

Cit.: DI:1. ARCo (1946), p. 119. Sense donar referència documental.

1366, desembre, 15. Barcelona.

Contracte del retaule de plata de Santa Maria de Salas. D'una part Pere Desvall de la tresoreria reial en representació del rei i Pere Oliver, canonge d'Osca, en representació de Santa Maria de Salas, i de l'altra Bartomeu Tutxó, argenter de Barcelona, signen les capitulacions per la realització del retaule. 
ACA, RP, Pergamins, carpeta 8, núm. 380.

Noverint universi quod die martis quintadecima mensis decembris anno a nativitate domini $\mathrm{M}^{\circ} \mathrm{CCC}^{\circ} \mathrm{LX}^{\circ}$ sexto, in presencia mei notarii infrascripti et Philipi Geraldi Rectoris Ecclesie de Salis prope Barbastrum diocesis Oscensis, Jacobi Munmany de domo domini Regis et Petri Constantini civis Osca testium ad hec vocatorum, venerabiles Petrus de Vallo, civis Barchinone, de Thesauraria domini Regis pro parte dicti domini Regis, Petrus Oliverii Canonicus oscensis pro parte beate Marie de Salis dicte diocesis oscensis, et Bartholomeus Tutxo argenterius Barchinone pro sui parte, et quilibet ipsorum, firmarunt, concesserunt et promisserunt servare et complere capitula que sequntur. Es avengut entre los honrats en Pere deç Vall per part del Senyor Rey, e en Pere Oliver per part dela Esglea de Santa Maria de Salas del bisbat d'Oscha, e en Berthomeu Tutxo argenter de Barchanona dela altra sobre les feadures del Rerataule que deu fer lo dit Berthomeu de manament del Senyor Rey ala esglea de Madona Santa Maria de Sales. Primerament que en Pere deç Vall per part del senyor Rey e en Pere Oliver per part dela esglea desusdita donen e paguen a present al dit Berthomeu Tutxo tres millia solidos barchinoneses a ops de comprar de present algun argent. Item li faran data de taula ab En Eymerich Dusay e ab en Jacme de Gualbes, cambiadors de Barchinona e pagadors com lo dit rerataule sia acabat de totes coses set mille solidos barchinonenses. E quels desusdits Pere deç Vall e en Pere Oliver, qui es reebedor dela decima antiga, prometen que si lo dit rerataulle muntava entre feadures e argent, a mes dits deu millia solidos, que ells li faran compliment tro empero a quantitat de dotze millia solidos ala qual quantitat entre fahedures e argent lo dit reretaulle no puxa sobrepujar, pero lo dit Pere Oliver hi sia obligat en tant com reebra res dela dita decima. Es encara avengut que si en lendemig, ans que lo dit rerataulle sia fet e acabat, lo dit Berthomeu per comprar mes argent haura mester una partida dels dits set milla solidos que lin puxa esser soltat tro en quantitat de IIII mille solidos i aço a coneguda den Pere Vites de casa del Senyor Rey. E lo dit Berthomeu Tutxo prometra que fara lo dit rerataulle be e complidament e acabada, e que sera fet sens tot embarach daçi per tot lo dia de Sent Johan de juny primer esdevenidor e per aço obligara lo dit Berthomeu tots sos bens mobles e seents sots pena de cent morabatins dor guanyadors al Senyor Rey quels haia a convertir en lo dit rerataulle. Item que haia per cascun march dargent obrat e daurat tot de fora aytant com la cara del altar sia, sis lliures devuyt solidos barchinonenses. Item que faça lo dit rerataulle ab la historia dels set goygs de Madona Santa Maria e que sien obrats dobra enlevada embotida ab bones figueres e ben cogitades. Item quel dit Berthomeu haia a pagar son or e totes les coses numerades al dit rerataulle en manera que ell haia acabat lo dit rerataulle a tota sa messio e despesa, salvo fust, mas ab totes clavadures. Item que com sia acabat lo dit rerataulle sia retut e donat per lo dit Berthomeu al dit Pere Oliver en presencia de Pere deç Vall o qui dells ha a cobrar apocha com la han 
rebuda, e no senç Pere deç Vall o qui ell volra. Quibusquidem capitulis, firmatis, concessis et promissis, predicti venerabiles Petrus de Vallo, Petrus Oliverii et Bertholomeus Tutxo requisierunt me notarium subscriptum ut de predictis facerem et conficerem duo publica instrumenta, de verbo ad verbum, consimilia, de quibus unum tradere dicto Petro Oliverii et aliud dicto Petro de Vallo, predictus vero Bertholomeus Tutxo $[\ldots]$ in posse notarii infrascripti predicta omnia attendere et compere sub obligacione omnium bonorum suorum habitorum et habendorum ubique ac sub pena in predictis capitulis contenta.Que sunt acta, concessa, promissa et firmata per partes iamdictas, Barchinone die et anno prefixis ac presentibus testibus supradictis ad hec specialiter convocatis.

Sig + num Petri de Bretonibus autoritate Illustrissi domini Regis Aragonum notarii publici per totam terram et dominacionem / suam qui predictis omnibus interfui. Et hec scripsi et clausi cum supraposito in linea XVa ubi dicitur solidos et.

1367, gener, 8 .

Pagaments al canonge Pere Oliver de 200 sous barcelonesos en concepte del viatge - que dura 22 dies- a Barcelona amb dues cavalleries i dos acompanyants, per contractar la realització del retaule de plata de Santa Maria de Salas amb l'argenter Bartomeu Tutxó, i a Zalema Avinganim, moro d'Osca, de dos cents sous per un mul $i$ un home que acompanyaren Pere Oliver en dit viatge.

ACA, RP, MR, vol 1803, fol. 31v.

Cit.: Del. ARCo (1946), p. 118. Sense donar referència documental.

Item posam en data los quals ${ }^{5}$ en Pere Oliver despes en .I. viatge que feu a Barchinona de manament del senyor Rey lo qual viatge lo dit Pere Oliver estec entre anar, estar e tornar XXII dies, ço es ab dues cavalcadures e dos homens de peu es assaber la I ${ }^{\text {a }}$ cavalcadura sua propia e l'altra de loger lo qual viatge feu per fer la avinença ab l'argenter que fahia lo dit retaule d'argent ${ }^{6}$.

Item posam en data los quals foren donats a Çalema Avinganim, moro habitador d'Oscha, per loger de .I. mul e .I. hom de peu que anaren ab lo dit Pere

\footnotetext{
${ }^{5}$ Segueix ratllat jo.

${ }^{6}$ Segueix ratllat CCXX solidos jaccenses.
} 
Oliver en lo dit viatge. E avi apocha feta e aclosa per en Pedro de Almança notari a VIII de janer del any dela nativitat de nostre Senyor M. CCCLXVII ${ }^{7}$-CC solidos jaquenses.

[Al marge]: Messions. E restitui letra del senyor Rey de manament als dits en Pere Oliver e Ramon Vigoros que anassen a Barchinona per aquests afers.

1367, març, 28. Saragossa.

Cfr. Document de 1364, abril, 23. Sant Mateu.

1367, maig, 25. Saragossa

Ramon de Vilanova "cavaller, conseller, tresorer del senyor Rey" davant l'incompliment per part de quatre canonges Pere Oliver i Ramon Vigorós dels terminis acordats de l'entrega dels diners per obrar el retaule de Santa Maria de Salas que el rei havia manat fer, i davant la proximitat de la finalització del retaule per part del mestre argenter a mitjans del mes de juny per tal de portar-lo a Osca el dia de la festivitat de Sant Joan Baptista -24 de juny-, ordena als dos canonges que dipositin les sumes necessàries a la taula d'Eimerich Dusay i de Jaume de Gualbes, canviadors de Barcelona, per tal que una vegada acabat el retaule aquests diners siguin entregats al mestre argenter.

AHC, Extravagantes-papel

Pub. par.: ESTEBAN (1993), p. 402.

[...] lo maestre del dit retaule ab sagrament e homenatge d'haver acabat aquell [...] que la moneda que sera necessaria al dit retaula sia deposada en la taula d'en Eymerich Dusay e d'en Jacme de Gualbes', cambiadors de Barchinona, ab condició que com aquell sera acabat, sia deslliurada al maestre que aquell fa.

${ }^{7}$ Segueix ratllat XLIIII solidos.

${ }^{8}$ ESTEBAN transcriu Servalbes. 
[1367, juny, 24, abans del]

Inscripció al dors del retaule de Santa Maria de Salas publicada per Francisco Diego de Aynsa el 1619.

Pub.: AYNSA (1619), p. 594.

Disl ARCo (1946). Anota que només pot llegir una part de la inscripció en els set compartiments conservats.

Aquest retaule ha fet de manement del senyor Rey en Pere Desvall de la trasoraria del dit Senyor a servey de la escleya de madona Santa Maria de Salas en esmena e satisfacció de cert nombre de lantes dargent, les quales o dit senyor feu pendre de la dita esgleya per mans del gobernador d'Arago per gran necessitat de la guerra de Castella com no hagues de que pagas los soldats, e per ço lo dit P. Desvall vene les dites lantes de manament del dit senyor, e distribuy la moneda per lo tresorer, ha procurat ab lo dit senyor quel dit retaule hi sia fet per ço suplica al senyor Vispe e als presberes de la dita esgleya que tots disautes sia dita una misa de la dona Santa Maria per lo senyor Rey e per la Senyora Reyna e que en remissio dos pecats sia feta commemoració del dit P. Desvall en satisfacció dels trevalls que ha sostenut en fer e percurar [que f]os fet lo present retaulo.

El retaule de plata obrat per Bartomeu Tutxó fou entregat al bisbe d'Osca i prior de Santa Maria de Salas que era també prior de Santa Maria de Salas.

Cit.: Estreban (1993), p. 400. Sense donar referència documental, tot $i$ que és de I’ACH.

1367, agost, 3. Saragossa.

El rei Pere mana al batlle del Regne d'Aragó i a les autoritats locals del bisbat d'Osca que deroguin les ordinacions que obligaven el bisbe d'Osca Jimeno de Ribavellosa a la construcció $i$ reparació dels murs de ciutats, viles $i$ poblacions. Aquest manament pot ser interpretat com a compensació de l'esforç que el bisbe havia fet en retornar els diners de la dècima que havia 
ocupat en altres afers en compte d'invertir-los en el retaule de Santa Maria de Salas.

ACH, sign. 2-276 i 2-179.

Cit.: Esteban (1946), p. 402.

Carta pública en què es fa constar el pes del retaule de Santa Maria de Salas obrat per l'argenter Bartomeu Tutxó. El retaule fou pesat en presència del notari de Barcelona Andreu Figuera. El pes fou de 94 marcs, 6 unces, 3 ternals $i$ mig de plata.

ACA, MR, RP, Pergamins Carpeta 8, núm. 384.

Cfr.: Document de 1368, febrer, 7. Osca.

1368, febrer, 7. Osca.

Albarà de tres mil tres cents trenta-tres sous i deu diners jaquesos que Ramon de Vilanova, cavaller, conseller i tresorer del rei, de manament del senyor Rei havia posat en l'obra del retaule de Santa Maria de Salas, per raó dels dos cents marcs de plata que el rei Pere havia pres de la dita església, per causa de la guerra amb Castella.

ACA, R.P. Pergamins Carpeta 8, núm. 382.

Sepan todos como yo don Per Oliver, canonge e arcidiagno delas valles en al seu dela eglesia de Jhesus Nazareno de la ciudat de Huesca e procurador que so del hondrado e discreto don Ramon Vigoros, canonge et arcidiagno de Santa Engracia de Çaragoça en la seu dela dita Ciudat a collir el segunto annyo dela decima bianal penultima atorgada al Senyor Rey por el Senyor papa Urbano quinto que agora yes en el dito vispado segunt parece por una letra de comision por el Senyor Rey feyta al dito don Ramon Vigoros e ami dada enla villa de Sant Matheu a XXIII dabril del annyo $\mathrm{M}^{\circ} \mathrm{CCC}^{\circ} \mathrm{LX}^{\circ}$ e quarto con carta publica de procuracion recebida por mi Joan Martinez de Sixona ${ }^{9}$ notario dius scripto en la ciudat de

\footnotetext{
${ }^{9}$ Sic, per Sixena.
} 
Caragoca a XX dias de deziembre en el annyo dela natividad de nuestro Senyor M.CCC.LX e VIII mas cerca pasado en la qual carta publica de procuracion yo he poder alas cosas dius scriptas segunt porel tenor dela procuracion paresce et ami notario dius scripto es cierto por aquella, por vigor dela procuracion et porel poder ami dado et atorgado en aquella et por mi mismo como comissario sobre dito, atorgo et recognosco avos muyt hondrado et discreto mosen Ramon de Villanova cavallero consellero et trasorero del Senyor Rey que havedes metido en aquel retaulo dargent que de mandamiento del Senyor Rey se es feyto para la esglesia de Santa Maria de Salas dela dita ciudat de Huesca por razon daquellos dozientos marcos dargent quel Senyor Rey se priso dela dita eglesia de Santa Maria de Salas, es asaber, tres mil trezientos trenta tres solidos et diez dineros jaqueses de los quales dineros por mi dito don Per Oliver solo [...] del dito mi conpanyero ende fue feyta avos dito trasorero otra apoca recebida en Huesca el ultimo dia doctobre del sobredito annyo mas cerca passado por en Per Bretons notario por toda la terra et senyoria del Senyor Rey, por mano del qual notario los sobreditos dineros fueron dedueytos et scriptos enel dorso dela sobredita comision ami et al dito don Ramon Vigoros, feyta de aquella mayor quantia de settecientas liuras jaquesas en la dita comision expresada. Los quales tres mil trezientos trenta tres solidos e diez dineros pagastes delos dineros del Senyor Rey que vienen en poder de vos dito trasorero a Bertholomeu Tucho, ciudadano et argentero dela ciudat de Barcelona porel qual el dito retaulo es seydo feto. El qual retaulo reconezco avos dito trasorero por mi et porel dito mi companyero del qual yo so procurador, que havedes enviado el dito retaulo ala dita eglesia de Santa Maria de Salas con el hondrado et discreto en Pere Dezval dela trasoreria del Senyor Rey, el qual retaulo es et esta en el altar de Santa Maria de Salas, que pesa Novanta quatro marcos seys onzas tres ternals et media dargent et aquel fue recebido apeso del dito argentero po en Per Vicent dela trasoreria del Senyor Rey segunt mas largament parece por carta publica reecebida en Barcelona a XV dias de setiembre del dito annyo mas cerca pasado por Handreu Figuera notario de Barcelona. Et poque vos dito trasorero aviedes puesto e pagado en el dito retaulo los sobre ditos tres mil trezientos trenta tres solidos et diez dineros. Et [have]des enviado el dito retaulo ala dita eglesia de Santa Maria de Salas enal qual es, quiero por mi et como procurador [...] sobredito que vos ende sia feyta la present carta publica testimonial por apoca de recepta delos ditos dineros e retaulo porel notario diuso scripto en testimonio delas sobreditas cosas pero priesto enel nopne qui desuso per [...] seer demandado al Senyor Rey pora la dita eglesia del residuo del dito argent acomplimiento delos ditos dozientos marcos quel Senyor Rey ende priso como el dito retaulo non pese tanto segunt era el dito argent. Esto fue fecho enla ciudat de Huesca; siet dias del mes de febrero, anno a nativitate Domini Millesimo trecentesimo sexagesimo octavo. Testimonios son desto Victorian Dartajona clerigo beneficiado en al eglesia dela dita seu de Jhesus nazareno, et Sancho Desparça, escudero, habitantes en la dita ciudat de Huesca. 
Sig + no de mi Johan Martinez de Sixena por auctoridat del Senyor Rey de Aragon notario general por toda la tierra e senyoria quin a las sobre ditas cosas present fue e aquest albara scribir fice dela nota del qual por mi recebida di e otro semblant albaran en publica forma al dito trasorero et el present al dito Per Oliver por razon dela presentacion porel feyta en aquel, con sobrescripto en la XVI ${ }^{a}$ linea ont se lie notarius.

[Verso]: [...] sobre lo fet de certa [...] dargent quel senyor Rey feu fer en la Egleya de Madona Santa Maria de Sales al bisbat dOscha per rao de 200 marchs dargent quel dit Senyor Rey se pres dela dita Esgleya en lany MCCCLXIIII per necessitat de la guerra de Castella.

1368, març, 1.

Pagament de 8000 sous jacquesos a Bartomeu Tutxó, argenter de Barcelona pel retaule de Santa Maria de Salas.

ACA, R.P MR. Vol. 1803, fol.31.

Cfr.: Doc. de 1366, octubre, 16. Barcelona.

1368, març, 1. Barcelona.

Àpoca de set mil sous de Barcelona per l'obra del retaule que Bartomeu Tutxó, argenter de Barcelona, havia obrat per a l'església de Santa Maria de Salas; la quantitat havia estat dipositada a la taula d'Eimeric Dusay $i$ Jaume de Gualbes, canviadors de Barcelona.

ACA, MR R.P Pergamins Carpeta 8, núm. 384.

Cfr.: També document de 1366, octubre, 16. Barcelona.

Cit.: DEL ARCO (1946), pp. 118-119. Sense donar referència documental.

Sepan todos como yo Bertholomeu Tucho, ciudadano et argenter dela ciudat de Barcalona atorgo haver havido et reecebido de vos hondrado et discreto don Per Oliver canonge et arcidiagno delas Valles en la seu dela eglesia de Jhesus Nazareno dela ciudat de Huesca asi como comisario que siedes deputado por el senyor Vispo 
dela dita ciudat de Huesca a collir el segundo annyo dela decima bienal penultima atorgada al Senyor Rey en el dito Vispado de Huesca porel Senyorpapa Urbano quinto que agora yes, yes a asaber diez mil solidos dineros jaqueses delos quales yo recibie por mano vestra los tresmil solidos en la dita ciudat de Barchalona et de aquellos vos fue feto por mi otro albaran et los siet mil solidos recibi por mano de Nameryc Dorray ${ }^{10}$ et de Jayme de Gualbes ciudadanos et canviadores dela dita ciudat de Barcelona en la taula delos quales vos posastes e metiestes los ditos siet mil solidos jaqueses pora dar a mi por razon de aquel retaulo dargent que de mandamiento del Senyor Rey yo havia a fazer pora la eglesia de Santa Maria de Salas dela dita ciudat de Huesca poral qual retaulo fazer vos diestes a mi los ditos tres mil solidos primeros et con aquellos me haviades a fer complimento alos ditos diez mil solidos delos ditos siet mil solidos por vos en la dita taula puestos, los quales siet mil solidos me fueron liurados por los ditos canviadores quando el dito retaulo que por mi feto ${ }^{11}$ liurado edado por mi por part del Senyor Rey al honrado en Pere Vicent, ciudadano dela dita ciudat de Barcelona e dela trasoreria del dito Senyor Rey pora enviar el dito retaulo ala dita eglesia, el qual peso novanta quatro marcos seis onças, tres ternals e medio dargent seunt parece por carta publica recebida en Barcelona a XV dias de setiembre del dito annyo mas cerca pasado por Andreu Figuera, notario de Barcelona, la qual carta fue recebida quando el dito fue por mi liurado al dito Pere Vicent, en presencia del qual el dito Andreu Figuera, notario et [de] los testimonios en la dita carta expresados fue pesado el dito Retaulo por el qual [...] delos ditos diez mil solidos yo recibie por part del muyt hondrado e discreto mosssen Ramon de Villanova, cavallero, consellero e.[trasorer]o del Senyor Rey dela quantia que del dito Senyor Rey venia en poder del dito trasorero, yes a saber [tres mil] trezientos trenta tres solidos e diez dineros jaqueses. E quanto delos ditos diez mil solidos que vos don Pere Oliver asi como comisario me haviades adar por el dito retaulo dela quantia que vos collides et recebides por el dito Senyor Rey dela sobre dita decima bienal, me atorgo seer bien pasado, contento et satisfeyto et pronto vos end [...]de danyo de de seer autor et defendedor si en algun tiempo vos sera feyta ni movida demanda ni question alguna delos ditos diez mil solidos por mi ni por alguna otra interpuesta persona dius obligacion de mis bienes. Et quiero que vos end sia feto el present albara por el notario dius scripto por tal que en testimonio de verdat lo trayades. Esto feu feyto en la ciudat de Barcelona dia miercoles, primeer dia del mes de março, anno a nativitate domini millessimo trecentesimo sexagesimo octavo. Testimonios son desto Guillem Çapera de casa del Senyor Vispo de la Ciudat de Huesca e Guillem de Seta, habitantes en aquella misma Ciudat.

\footnotetext{
${ }^{10}$ Sic, per Dusay.

"Segueix interlineat el qual retablo fue.
} 
Sig + num de mi Johan Martinez de Sixena por autoridat del Senyor Rey de Aragon notario general por toda su tierra e senyoria qui a las sobre ditas cosas present fue e aquest albara scrivir ficie con rasos e en mendados en la primera linea

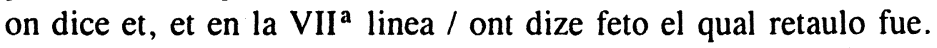

[verso]: Apocha dels VII mille sous del retaule pagats an Bertomeu Tuxo argenter. Et con un albaran que ha dentro de aquest de III mille solidos. Resta... X mille solidos.

1381, juny, 4. Saragossa.

Dubtes dels comptes relatius al retaule d'argent de Santa Maria de Salas inclosos entre els dubtes de la collita de la dècima del bisbat d'Osca que el papa havia atorgat al rei Pere el Cerimoniós.

ACA, RP. MR. Col 1803, fols. $48-49 v:^{12}$

Item part los deutes dessusdits ha en lo present compte los dubtes e defalliments deiusdits $[\ldots]$

Item en XXX cartes fa a deliberar si los seran preses en compte CCL solidos que poien haver despeses en messions per perseguir quel senyor Rey lus encartas els faes assignació del argent de Santa Maria de Salas car no par quel senyor Rey acostum de pagar semblants messions o al menys quen hagen manament al Racional quels sien rebudes en compte. E encara falria compte per menut a la dita data e apocha de .I. hom que diu quen trameteren a Sant Matheu. Fo acordat per lo Racional que la dita data sia moderada cor quel dit compte per menut e apocha ho han ne porien haver en alguna manera. Apres fo acordat per lo dit Racional que y sien preses en compte $\mathrm{CC}$ sol. E axi fo fet perque fo [...]

Item en XXXI cartes a la data de Xm solidos jacquenses que posen haver feta an Barthomeu Tutxo argenter per obra del retaule son los dubtes seguents: primerament que par que la dita quantitat deia esser de barchinonesos e no pas de jaquesos, jassia ells mostren apocha de jaquesos. E parria manifestament que sia estada error del escriva qui feu l'apocha per les rahons seguents. Primerament car si es feta mencio quel dit argenter reebe de Ia part IIIm solidos jaquesos de quen feu

\footnotetext{
${ }^{12}$ Sense donar cap referència documental. DEL ARCO (1946), p. 119 esmenta que a l'ACA consta el detall de la dècima de l'any 1367 recaptada pels col-lectors a la diòcesi d'Osca amb destinació al retaule de Santa Maria de Salas. Es tracta del volum 1.803 del RP. MR.
} 
apocha la qual apocha ells monstren e no es ver que sia de jaquesos ans de barchinonesos.

Item hi es feta mencio quels romanents VIIm solidos jaquesos foren posats per en Pere Oliver en la taula den Eymerich Dusay e den Jacme de Gualbes e acabat lo reetaule foren dats al dit argenter a compliment dels $\mathrm{Xm}$ jaquesos que devia haver. E apar per covinença de que y ha carta publica quels dits Xm solidos a ell deguts dels quals eren los dits VIIm quis posaren en la taula e los IIIm primers devien esser barchinonesos e no jaquesos perque en alguna manera ell no diria ne podia pagar sino de barchinonesos. E deuen restituir cauteles necessaries a la dita data. Dix lo dit en Ramon Vigoros qui fo present a la determinacio dels presents dubtes e encara en Pere Rodellar prevere qui per part dels hereus del dit en Pere Oliver fo aximateix present a la dita determinació que cert es e ver que los dits $\mathrm{X}$ mille solidos foren barchinonesos e no pas jaquesos perque fo adobada la dita data ço es que fo mudada la quantitat de barchinonesos mas fan hi a cobrar cauteles.

Item per que degen restituir certificacio den Pere dez Vall que per la dita raho no haia pagat al dit argenter per part del senyor Rey com lo dit Pere dez Vall si fos obligat ensemps ab lo dit Pere Oliver segons apar per la dita covinença. Acorda lo Racional que la dita certificació no es necessaria car si res per la dita raho hagues pagat lo dit en Pere dez Vall en la letra debitoria de les DCC lliures jaqueses feta per lo senyor Rey per l'argent que pres de Santa Maria de Sales ne fora estada feta deduccio. E apar per la dita carta la qual es en poder del Racional que noy ha deduccio alguna per la dita e mas i maior cautela sien regoneguts los libres de notaments del ofici si se hy trobara notament algun.

Item deuen esser demanats de compte los dits Pere Oliver e Ramon Vigoros dela obra del dit reetaule. En lo qual compte deuen posar la rebuda los dessusdits $\mathrm{Xm}$ solidos o tant com asin posaran en data. Item hi deuen posar en rebuda IIIm CCC XXX III solidos X diners jaquesos quel dit Pere Oliver reebe del tresorer par convertir aquells en la dita obra segons quen es feta mencio en .I.a carta publica que es en poder del Racional e encara apar per la deduccio que la dita quantitat es estada feta en lo dors dela letra debitoria de DCC lliures jaqueses feta per lo senyor Rey en satisfaccio del argent que havia pres de Santa Maria de Sales la qual letra deu esser vista en la recepcio del dit compte ensemps ab la carta dela covinença dessusdita feta entre en Pere dez Vall e en Pere Oliver e lo dit argenter per ço que sia vist quel dit reetaule sia fet segons que era ordonat per lo senyor Rey e aximateix per veure les deduccions escrites en la dita letra deles pagues ques son fetes del dit dute.

Item es feta mencio en la dita letra debitoria quen Blasco Aznarez de Borau batle general d'Arago reebe per part dela Cort CC marchs d'argent quel senyor Rey pres dela dita esgleya de Santa Maria de Sales per los quals encarta les dits DCC 
lliures per que fa a demanar del compte del dit argent lo dit Blasco si recur nol ha $^{13}$.

\section{BIBLIOGRAFIA}

AYNSA Y DE YRIARTE, Francisco Diego de (1586-1628), Fundación, excelencias, grandezas y cosas memorables de la antiquissima ciudad de Huesca, assi en lo temporal como en lo espiritual: divididas en cinco libros...; Recopiladas por Francisco Diego de Aynsa y de Yriarte... En Huesca: Por Pedro Cabarte, 1619.

FACI, Fray Roque Alberto de, Aragón, Reyno de Christo y dotes de Maria Santissima fundado sobre la columna inmobil de Nuestra Señora en su ciudad de Zaragoza, aumentado con las apariciones de la Santa Cruz, Santissimos Misterios, Milagros del Smo. Sacramento, Imágenes de Cristo N.S. y con los aparecidos, hallados, antiguos y milagrosos de. En el Mismo Remo Zaragoza. En la Oficina de Joseph Fort enfrente del Colegio de San Vicente Ferrer, 1739. Imprenta Francisco Morena, 1750.

DE ZARAGOZA, Lamberto (OFM Cap) 1711-1785, Teatro historico de las iglesias del reyno de Aragon...; su autor el P. Fr. Lamberto de Zaragoza. En Pamplona: en la imprenta de la viuda de don Joseph Miguel de Ezqueno, 1780-1802, 9 v. Els últims cinc vols. són de HuEsCA, Ramón de.

QuAdRAdo y NieTo, José María, Aragón, por José María Quadrado, Barcelona, Cortezo, 1886. Colección "España, sus monumentos y artes, su naturaleza e historia".

BERTAUX, Emile, L'exposition retrospective d'Art à Saragose 1908. Introduction de M. Paso y Ruata. Préface de Paul Moreno. Saragosse et Paris, 1910.

Exposición retrospectiva de Arte 1908, organizada bajo las auspicias del excelentisimo Arzobispo de Zaragoza, por la Real Junta del Centenario de los Sitios, Zaragoza, 1808-1809.

\footnotetext{
${ }^{13}$ Segueix ratllat sia lin fet.
} 
BorRÁs GuAlis, Gonzalo M. Enciclopedia temática de Aragón. Tomo 3. Historia del Arte I: De la Prehistoria al fin de la Edad Media. Director y autor Gonzalo M. Borrás Gualis, Zaragoza, Monayo, DL, 1986-1987.

\section{RÉSUMÉ}

Le propos de cet article est strictement documentaire. Son objet c'est de donner à connaître quelques documents inédits -quoique, dans certain cas, son contenu était déjà connu - et grouper les donnés conservés aux Archives sur le rétable de Santa Maria de Salas (Huesca, Aragon) afin d'offir un premier "corpus" documentaire.

\section{SUMMARY}

The aim of this article is strictly documentary. Its object is to show some unpublished records - although, in some cases, their content was known- and join together a group of data obtained from the Archives about the retable of Santa Maria de Salas (Huesca, Aragon), in order to offer a first documentary "corpus". 